\title{
Cell biology: delivering tough cargo into cells
}

\author{
Vivien Marx
}

New approaches expand the range and size of materials that can be inserted into a cell.

Ferrying foreign material into cells is routine in many labs, and with many cell lines its efficiencies are often high. Scientists can choose from a wide menu of approaches including vector-based methods; chemical approaches with liposomes, polymers or cell-penetrating peptides; physical manipulations such as microinjection; and the use of voltage with electroporation.

Some cargo types-such as longer pieces of DNA or RNA, peptides, proteins, antibodies, and nanoparticles-are difficult, often impossible, to get into cells, especially primary cells. And even if the cargo transfer is successful, often too many cells die afterwards. Cells can chew up foreign DNA. The delivered cargo may arrive and be endocytosed but then be degraded instead of escaping the endosome. "The cell likes to regulate what it takes in and out, and it really doesn't want everybody else to tell it what should go across that membrane," says Massachusetts Institute of Technology (MIT) chemical engineer Klavs Jensen.

Jensen and others are developing ways to bring cargo such as large biomolecules or nanoparticles intact across a cell membrane, to the right address and with the assurance that the cargo will be functional after its journey. It's a delicate challenge. "It is difficult to generate a large-sized pore on a cell without killing it," says University of California, Los Angeles (UCLA), biomedical engineer Eric Pei-Yu Chiou. And the pores open only transiently and reseal quickly.

Tools are lacking for transfecting supersized cargo such as whole bacteria or mitochondria, says Chiou. Proteins, enzymes or conjugated quantum dots are slightly smaller, but labs tend to underestimate the difficulty of delivering slowly diffusing molecules through a transient opening in the cell membrane.

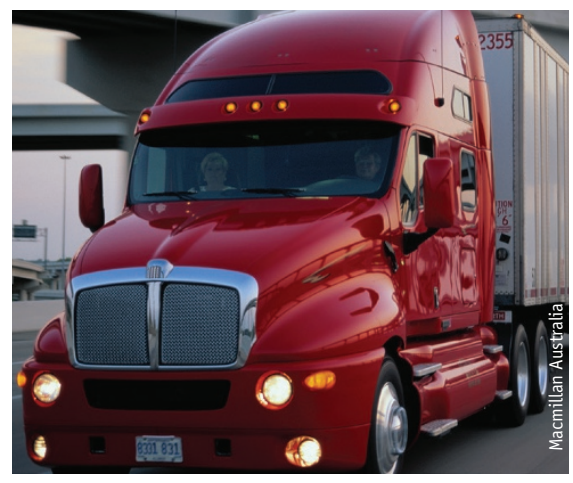

Some cargoes are too tough to transfer into cells. Methods developers say emerging approaches can handle more difficult cargo.

In addition, different cargo types have varying levels of cell toxicity, says Haoyi Wang, a genomics researcher at the Institute of Zoology at the Chinese Academy of Sciences in Beijing, who also has an appointment at The Jackson Laboratory. In each case a balance must be struck between transfection efficiency and impact on cell viability, he says.

This balance matters greatly as labs explore new possibilities and also with a view to cell-based therapies. Cargo transportation issues can arise when labs generate gene-edited transgenic animals, build cell-based assays for large-scale screens with many cargo types, or experiment with traditionally finicky cells such as neurons, immune cells and stem cells. Some cargoes are tough. Or, as some methods developers say, the cargo is just too tough for classic delivery methods.

\section{CRISPR cargo}

Bacteria ward off infection with the help of the immune mechanism called clustered, regularly interspaced short palindromic repeats (CRISPR) and CRISPR-associated proteins (CRISPR-Cas). This mechanism has been turned into a gene-editing tool in which the Cas9 nuclease serves as molecular scissors and specialized RNAs guide Cas9 to the specific spot in the genome for the intended edit. One challenge is that CRISPR reagents are relatively voluminous, creating a bottleneck for the field, especially with in vivo delivery.

For cells in culture, plasmids can deliver the entire CRISPR-Cas9 package, but the load challenges intensify for gene editing in vivo, which is also relevant for somatic gene therapy, says Wolfgang Wurst at Helmholtz Zentrum in Munich. Viral vectors such as lentiviruses or adenoviruses can serve as delivery vehicles, and all the CRISPR-Cas reagents fit on one vector. But, says Wurst's colleague Oskar Sanchez Ortiz, the disadvantage is a potentially strong immune response. And insertional mutations can occur when the lentivirus integrates into the genome.

Adeno-associated viruses, which are favored for gene therapy, do not induce such immune responses. But their cargo-carrying limit is around 5 kilobases. Actually, says Ortiz, the limit lies between 4.5 and 4.8 kilobases, which is better for virus production. The CRISPR load adds up: the full Streptococcus pyogenes Cas9 (SpCas9) coding sequence accounts for $\sim 4.5$ kilobases; added to that are the promoter and terminator sequence and the guide RNA with its own promoter sequence. Altogether, says Ortiz, the sum of these components exceeds 5 kilobases.

Some researchers try to reduce the size of the CRISPR reagents package by, for example,

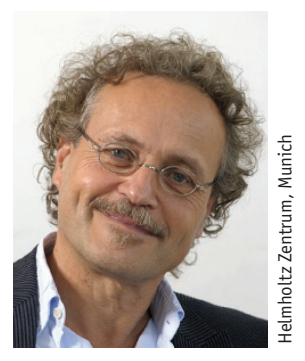

Gene editing in vivo intensifies cargo load challenges, says Wolfgang Wurst. 
removing parts of Cas9. But, says Wurst, these steps can crimp enzyme activity. Labs are exploring proteins that are similar to Cas9 but smaller, but these are not yet as well characterized as Cas9. And, he says, their protospacer-adjacent motif (PAM), which is the sequence next to the one that Cas 9 targets, can be more complicated than the one that Streptococcus pyogenes' Cas9 needs. Given these issues, and the

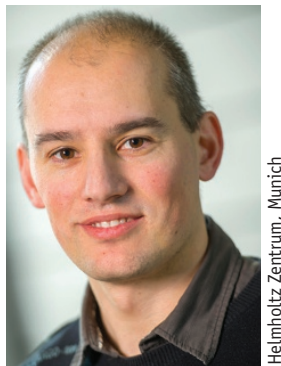

fact that Cas9 is the best-characterized nuclease in terms of its structure and offtargets, he and Ortiz decided to work on a Cas9-oriented step to address cargo loading.

The structure of Cas9 is a sandwich of sorts, with two nuclease domains. Wurst and Ortiz decided to split this sandwich to obtain cargo in line with A AVs' carrying capacity $^{1}$. They use are between 4.5 and 4.8 kilobases, which exceeds the cargo-carrying limit of adeno-associated viruses, says 0skar Sanchez Ortiz. two different AAVs. Each of the Cas9 parts is independently expressed by one AAV, and each Cas9 part is fused to a split intein, which splices the Cas9 parts together.

When the two split inteins bind to one another, the process that unfolds is similar to the RNA process of exon-intron splicing. The two Cas9 parts are fused to become a single protein that functions like wildtype Cas9. This split-Cas9 system lets the researchers control the expression of each half using different promoters, and it could allow researchers to direct Cas9 activity to a very specific cell population.

Several other labs are splitting Cas9 for transport and to enable more precise tuning of the protein's activity, including Jennifer Doudna and her team at the University of California, Berkeley, and Feng Zhang and his team at $\mathrm{MIT}^{2,3}$.

Zhang and colleagues have described Cpf1, which, unlike Cas9, appears to have a single nuclease domain ${ }^{4}$. Although the authors note it might contain a second, "yetto-be-identified" nuclease domain, the RNA associated with Cpf1 is much shorter.

Other approaches involve conjugating Cas9 protein and the guide RNA to a cellpenetrating peptide, which is then delivered to cultured mammalian cells.
A group at Seoul National University took recombinant Cas9 and combined it with guide RNA to create a ribonucleoprotein (RNP). The authors note that this RNP approach leads to quick edits and avoids the risk of unwanted plasmid insertion into the genome. These RNPs degrade quickly in the cell, which reduces the risk of off-target effects. A separate approach developed at Harvard University and labs at several other institutions involves packaging the CRISPR reagents in a cationic lipid formulation. And a group at University Medical Center Utrecht and their colleagues developed an active uptake method called induced transduction by osmocytosis and propanebetaine (iTOP) to deliver native proteins, including Cas9 and other molecules, into cells.

\section{Scaled up in vivo transfer}

"The nice thing about electroporation is that one can deliver very large vectors," says geneticist Ken Scott at the Baylor College of Medicine (BCM). In his lab, transfection with DNA vectors in cell culture is routine. He does not run into load-bearing constraints in those experiments, in which he uses lentivirus as the vector for nucleic acids for high-throughput screening and which he is scaling up to use for in vivo screening in mice. To do this, he applies a dual piggyBac system, originally discovered in baculovirus, in which a mobile genetic element, a transposon, helps with cargo delivery.

The advantage of this system, says Scott, is that it lets researchers express the transposase from one vector, which then allows integration of the DNA carrying a gene of interest under the control of a lineage-specific promoter that is located on the other vector. "The transgene can, in theory, carry anything," he says, with some labs showing it can carry 100-kilobase loads and perhaps as much as 200 kilobases. The team is still exploring how many cells actually take up the DNA, but, he says, "my sense is it's a lot, particularly as one increases vector amount."

Scott and his BCM collaborator Ben Deneen have also been delivering cargo directly to mouse embryo brains and, with the help of surgical techniques, to adult mouse pancreas. They electroporate the following cargo directly into the pancreas: a vector that expresses transposase, the enzyme that helps a transposon move from one location to another; a piggyBac transgene carrying their gene of interest; and Cre recombinase, an enzyme that directs recombination, both expressed via a pancreas-specific promoter, which activates the oncogenic KRAS ${ }^{\mathrm{G} 12 \mathrm{D}}$ in their model. With this approach, the researchers can activate genes associated with metastasis along with a fluorescent marker gene enabling them to characterize physiological processes such as increased metastasis.

A team at MIT, Tufts University and Harvard Medical School used hydrodynamic injection of a plasmid encoding Cas9 and its guide RNAs into mice, to target specific tumor-suppressor genes in as many somatic cells as possible ${ }^{5}$.

Other teams deliver CRISPR-Cas reagents in vivo in a scaled-up approach also designed with a view to other types of cargo ${ }^{6,7}$. Microinjecting one CRISPR reagent into one zygote at a time is a slow way to create genetically modified animal models. "You need a good micro-injectionist with years of experience to do this," says Wang. Success rates vary with the genome target and mouse strain, but live-birth rates hover around $30 \%$.

To transport CRISPR-Cas9 components-including Cas9 mRNA, guide RNA and the donor oligonucleotide-more efficiently into zygotes, Wang and his team electroporated 50-100 zygotes in each cuvette. Compared to microinjection, they doubled the live mouse birth rate. Now,

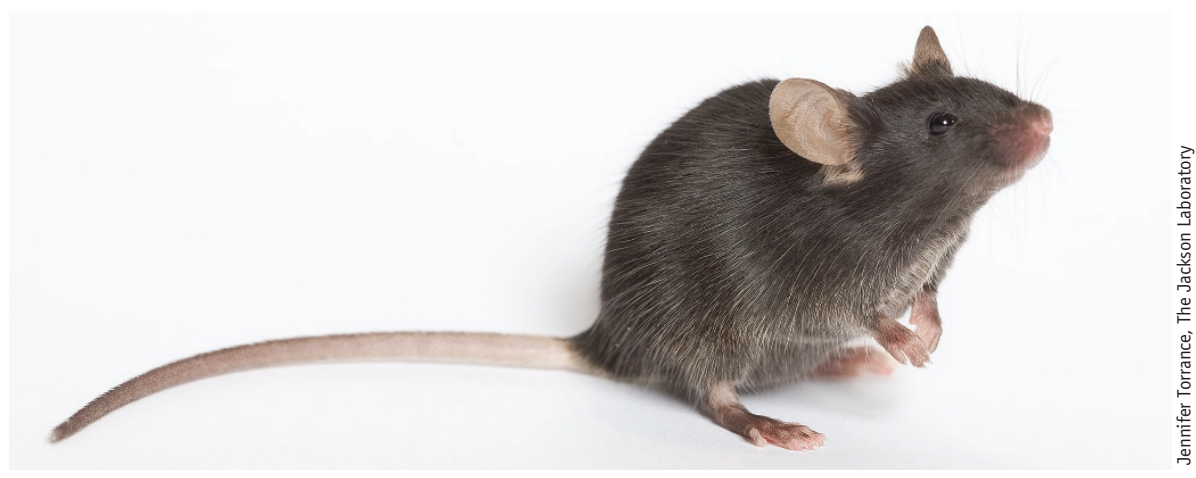

Some teams explore ways to deliver CRISPR-Cas reagents in vivo in a scaled-up approach. 
the researchers are testing this protocol on 10-20 gene candidates and comparing electroporation to microinjection.

The team tried lipofection alongside electroporation but decided to pursue electroporation with very low voltage, says Wang. Their electroporation approach can be used to deliver several types of gene-editing reagents, and they have delivered mRNA for enhanced green fluorescent protein as well as gathering some experience with the delivery of proteins.

The gene-editing machinery with CRISPR is not a "game changer" for cargo delivery, says Rodolphe Barrangou, a researcher at North Carolina State University. But ongoing CRISPR research efforts highlight a shift away from packaging long Cas9 genes and guide RNAs into viruses and toward the use of mRNAs or RNPs as delivery options.

These latter delivery approaches, says Barrangou, are convenient and useful because they offer transient function without long-term impact on cells and they avoid the risk of leaving exogenous DNA behind in the genome of the edited cell. Barrangou is also on the board of directors at Caribou Biosciences and co-founder of Caribou spinout Intellia Therapeutics. Both companies are developing CRISPR-Cas9-based therapies, also in a partnership with Novartis.

\section{Booming and bending}

Some labs work on general-purpose delivery approaches for large cargo. UCLA bioengineers Eric Pei-Yu Chiou and Michael Teitell wanted a better large-cargo transfer approach for experiments involving somatic cell nuclear transfer, in which an embryonic stem cell is made by removing the nucleus from an oocyte and replaced with an injected nucleus from a somatic cell.

The team's biophotonic laser-assisted surgery tool (BLAST) cuts semicircular openings in the cell membrane with flow created by exploding bubbles ${ }^{8}$. The system uses positive pressure to drive cargo into the cell. Cellular self-repair quickly seals these openings, such that diffusion would not provide enough time for large-cargo delivery, says Teitell.

BLAST can deliver cargo to hundreds of thousands of cells at a time. Cells are grown on a platform pocked with holes. Each hole is an entry to a fluid-filled channel through which cargo can be delivered. The tip of each hole has a crescent-shaped metal coating, and when a laser scans over the holes, the metal is heated and bubbles form.
Electroporation usually involves nanometer-sized cargo, but BLAST can work with larger cargo. The team has delivered into cells a diverse set of cargoes up to $3 \mu \mathrm{m}$ in size: an active enzyme, mitochondria, bacteria, dyes, beads and polymer-conjugated quantum dots. "The process is tunable for each cell type and cargo type to be transferred, making it a robust and highly adaptable approach," says Teitell. The team has used the technique successfully on challenging cell types such as human pluripotent stem cells, primary cultured mouse neurons and nonadherent cell types. They are now tackling mouse and human lymphocytes, which are notoriously difficult to transfect.

Making a 3- $\mu \mathrm{m}$ hole in the cell membrane with traditional methods such as laser cutting will kill the cell, because too much membrane material is lost, says Teitell. Their approach is more like a "cat door" that swings open and then likely swings shut once the cargo is delivered and the fluid stream ceases. The researchers do not know this for sure because the process is challenging to capture in real time.

The platform is a scaling-up of the engineers' photothermal nanoblade - a glass capillary with an outer metallic coating. When this nanoblade touches the cell membrane, heat and bubbles can explode holes in the cell membrane. One of their ongoing nanoblade projects is large-cargo transfer with single cells. They have delivered plasmids for green fluorescent protein through the plasma and nuclear membranes simultaneously, says Teitell.

With BLAST, the team has been able to increase throughput of large cargo delivery from 100 cells an hour to 100,000 cells in ten seconds. They have launched a start-up called NanoCav to turn the technology into a marketed instrument. Teitell believes that the range of potential applications is expanding from cell and genome engineering to organelle transfer and the dissection of intracellular pathogen life cycles and drug targets.

\section{Tough cargo to tough cells}

Often the cargo transfer process is toxic to cells, or the delivery fails. It's doubly challenging to deliver difficult cargo to finicky primary cells such as lymphocytes. With these types of experiments, researchers will want to optimize both the transfer method and the cargo itself, says Andrea Toell, product manager at Lonza Pharma\&Biotech Bioscience Solutions. Researchers should test the substrate's purity and stability and

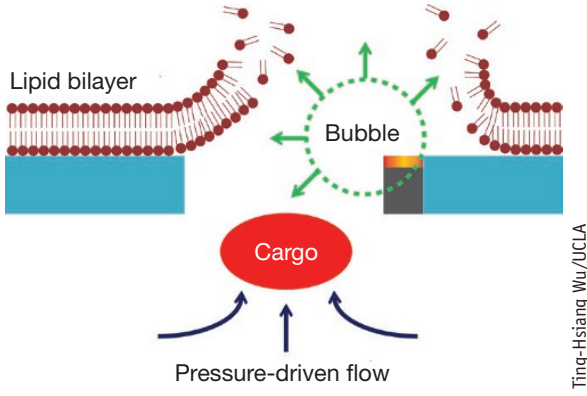

BLAST delivers cargo into cells with the help of exploding bubbles.

titrate the optimal cell-to-substrate ratio; and when working with mRNA, she advises that it should be capped and polyadenylated. Nucleofection is the company's improvement on electroporation, introduced as a nonviral transfection method for primary cells and hard-to-transfect cells, she says. The company developed a spectrum of protocols and voltage parameters for specific cell types and a range of cargo types.

MIT chemical engineers Klavs Jensen, Robert Langer and their then graduate student Armon Sharei developed a delivery method in which cells are squeezed as they travel through an array of channels with constricted sections ${ }^{9}$. The forces acting on the cells moving through the strictures create a transient hole in the cell's membrane. "Anything that is outside can go through these holes into the cell as long as your cargo is smaller than the hole," says Sharei.

In testing many cell lines, the group realized that the platform's performance extended from dyes with subnanometer dimensions to nanoparticles and carbon nanotubes several nanometers in diameter, says Jensen. Having a general-purpose platform to mechanically deform cells can help labs avoid the need for an array of tools for different cargo types and sizes.

Sharei has since completed a postdoctoral fellowship and in February 2015 became the full-time CEO of SQZ Biotechnologies, a company he co-founded with Jensen and Langer to commercialize this platform. The company eyes cell engineering for therapeutic purposes as well, and it collaborates with academic labs but also increasingly with biotech and pharmaceutical companies.

In a recent collaboration with researchers at MIT and Harvard Medical School, the team delivered siRNA into several types of mouse and human immune cells, including $\mathrm{T}$ and $\mathrm{B}$ cells. Although electroporation worked and achieved similar knockdown levels, the cells differed biologically: cells 
into which siRNA had been delivered showed better survival in the microfluidic device. The team also ferried a protein into B cells. "B cells are notoriously difficult to transfect by any other means and proteins are also notoriously difficult to get in with any of the conventional means, "says Sharei.

Chemistry and physics can help explain why some cell types resist the transfer of large molecules, says Sharei. Transfection techniques can succeed with nucleic acids because they are negatively charged. But classic transfection methods can fail in the wide world of less charged structures such as proteins, peptides or small molecules. From a charge perspective, a DNA plasmid is quite unlike an antibody.

The failure of classic transfection methods is a rather taboo subject, says Sharei. Some cell types, such as human-derived T cells, "really hate" certain transfection methods, he says. Experiments with nanoparticles often fail, and electroporation is frequently toxic for cells. But labs do not usually pres-

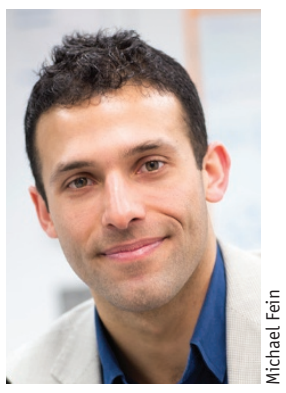
ent electroporation data along with gene expression data about their transfected cells and controls. Sharei, too, has omitted these experiments, because "you almost don't want to know" the degree of perSome cell types, such as human-derived T cells, just despise certain transfection methods, says Armon Sharei. could be a big service to everyone," he says.

Wang agrees that electroporation likely causes gene expression changes, but so does any kind of cell membrane disruption. "Poking a hole in a cell is going to definitely cause a lot of gene expression changes," he says. Yet these are likely to be transient changes. To check on any negative impact a transfer method may have, for example with in vivo experiments, Wang recommends that researchers investigate whether embryo development is impaired.

In Lonza's labs, Toell and her colleagues assess the potential impact of electroporation by looking at cell morphology and the functionality of pathways and markers, such as pluripotency markers with induced pluripotent stem cells. They have not done comprehensive studies on the impact of electroporation on gene expression. But some time ago, they compared nucleofection and lipofection and looked at upregulation of interferon-related genes after transfection with siRNA, and they did not see any significant effect of nucleofection, she says.

A number of groups now intently study the effect of electroporation on cells, says Jensen. He, his team and the Langer lab are looking into the basic biology and physiology that unfurls when a cell is rapidly and mechanically deformed. This research can help characterize the size and number of holes that delivery method will create. And it will offer insight about gene expression changes and biochemical pathways that are activated as the hole is created. Jensen and his team genetically knock out different cellular pathways and use inhibitors to systematically explore the steps involved in opening and repairing the cell membrane.

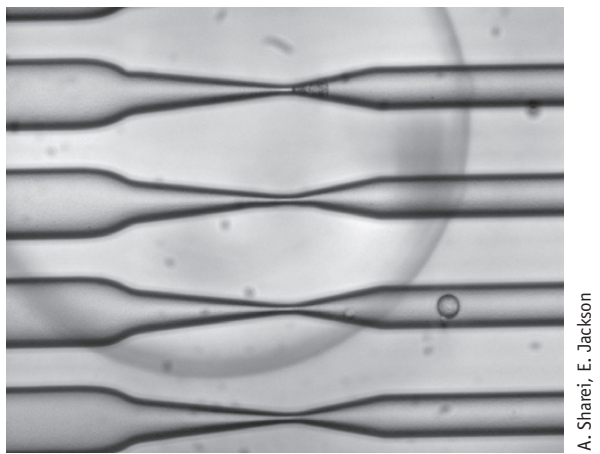

One delivery method squeezes cells as they travel through an array of channels with constricted sections, which leads to a transient hole in the cell's membrane.

With an understanding of the basic biology, scientists can explore "an even better way of delivering that cargo to the right address," says Jensen. That better understanding, both of the classic and newer cargo transfer methods, will help labs optimize delivery approaches for all sorts of tough cargo.

1. Truong, D.-J.J. et al. Nucleic Acids Res. 43, 6450-6458 (2015).

2. Wright, A.V. et al. Proc. Natl. Acad. Sci. USA 112, 2984-2989 (2015).

3. Zetsche, B., Volz, S.E. \& Zhang, F. Nat. Biotechnol. 33, 139-142 (2015).

4. Zetsche, B. et al. Cell 163, 759-771 (2015).

5. Xue, W. et al. Nature 514, 380-384 (2014).

6. Qin, W. et al. Genetics 200, 423-430 (2015).

7. Kaneko, T. et al. Sci. Rep. 1, 6382 (2014).

8. Wu, Y.-C. et al. Nat. Meth. 12, 439-443 (2015).

9. Sharei, A. et al. Proc. Natl. Acad. Sci. USA 110, 2082-2087 (2013).

Vivien Marx is technology editor for Nature and Nature Methods

(v.marx@us.nature.com). 